\title{
Direct Inguinal Hernia
}

National Cancer Institute

\section{Source}

National Cancer Institute. Direct Inguinal Hernia. NCI Thesaurus. Code C34691.

A protrusion of the abdominal cavity contents into the inguinal canal through the superficial inguinal ring. 